\title{
Desenvolvimento de uma plataforma multimídia como objeto didático para compreensão dos eventos da gênese dentária
}

Fellipe Moraes*; Tatiane Magalhães Alves**; Tacio Freire Silva***; Carla Figueiredo Brandão****; Jane Luzia Freire Matos*****.

\section{RESUMO}

A tecnologia tem se difundido gradativamente em todas as esferas da sociedade, se estabelecendo como indispensável. No âmbito da educação não é diferente, ela vem abrindo novas portas para o desenvolvimento do conhecimento. O presente trabalho tem como objetivo descrever a, elaboração de uma plataforma multimídia sobre gênese dental e as possíveis alterações dento-alveolares que dela se originam, para que os estudantes tenham uma visão ampliada, dinâmica e interdisciplinar deste conhecimento. Como referencial teórico foram utilizados livros que servem como base no ensino da odontologia, além de buscas de artigos científicos nas bases de dados Scielo, PubMed, Periódicos CAPES, e em outras fontes de informação, como o Google Acadêmico. Para a elaboração do instrumento foi utilizado o sistema Flash + ActionScript 3 (AS3) na plataforma Adobe AIR, permitindo ao aluno a navegação em todas as fases formativas do elemento dentário à medida que clicarem no
Cirurgião-dentista graduado pela Escola Bahiana de Medicina e Saúde Pública (EBMSP)

Graduanda em Odontologia pela Escola Bahiana de Medicina e Saúde Pública (EBMSP)

* Graduado em Sistemas de Informação pelo Centro Universitário da Bahia (FIB)

Mestre em Odontologia, Professora Assistente da Escola Bahiana de Medicina e Saúde Pública (EBMSP) Doutora em Radiologia Odontológica, Professora Adjunta da Escola Bahiana de Medicina e Saúde Pública (EBMSP)

ambiente desejado. A interdisciplinaridade e interatividade dos assuntos permitidos por esta plataforma oportunizará ao discente a construir um conhecimento de alta relevância na odontologia, servindo de instrumento didático dentro da nova tendência do ensino que, consequentemente, ajudará no raciocínio da investigação do diagnóstico.

Descritores: Educação. Software. Odontologia. Tecnologia. Aprendizagem. Anomalia.

\section{INTRODUÇÃO}

A tecnologia tem se difundido gradativamente em todas as esferas da sociedade, se estabelecendo como algo indispensável e promovendo evolução.

No âmbito da educação, não é diferente, a tecnologia tem aberto novas portas para o desenvolvimento do conhecimento, trabalhando aquilo que já é conhecido de uma maneira inovadora e instigante. 
Desse modo, a incorporação de novos recursos na educação amplia as condições do aprendiz para descobrir e desenvolver suas próprias potencialidades, permitindo que $\mathrm{o}$ mesmo possa progredir intelectualmente ${ }^{1}$.

De acordo com $\mathrm{Melo}^{2}$, a educação não deve se basear apenas na instrução repassada pelo professor, mas na construção do conhecimento na dialética aluno/professor e no desenvolvimento de novas competências, o que pode ser alcançado através da motivação dessa geração, por intermédio das ferramentas digitais.

Dentro desse contexto, a utilização dos softwares didáticos como objeto de informação permite maior interatividade na abordagem dos conteúdos $^{3}$, se tornando um ótimo aliado ao novo conceito de ensino-aprendizagem, em que o professor deixa de ser o transmissor de conhecimentos e passa a ser um facilitador ${ }^{4}$.

Conforme Vogel e Klassen ${ }^{5}$, este novo mecanismo de ensino transforma o papel dos alunos de receptores passivos de informação para participantes ativos, capazes de transformar e ampliar o seu conhecimento.

$\mathrm{O}$ que pode ser mediado pelos recursos digitais, que têm apresentado nos últimos anos um impressionante desenvolvimento, culminando na crescente utilização destes objetos no ensino acadêmico ${ }^{6}$.

Reynolds $^{7}$ destacou que a implementação dos avanços tecnológicos na educação odontológica, a exemplo do Ambiente Virtual de Aprendizagem (AVA), da IVIDENT (Escola Dental Virtual), da UDENTE (Universal Dental E-learning), influenciou de modo positivo no desenvolvimento de novas técnicas de ensino, se apresentando como uma excelente oportunidade na educação superior.

De acordo com Niederhauser e Stoddart $^{8}$, os computadores podem ser utilizados como catalisadores para estimular o movimento de reforma educacional, se apresentando como grande promessa para melhorar a instrução, contudo, em última análise, os professores é que iriam determinar o potencial da sua utilização.

Haguenauer ${ }^{4}$ corrobora com o trabalho citado anteriormente, pois segundo ela, apesar de todas as facilidades e vantagens atribuídas a tecnologia, a metodologia e a pedagogia assumem o papel principal no processo ensinoaprendizagem, de modo que o professor é o principal responsável pelo êxito do programa de ensino, tendo como papel primordial nesse novo modelo a escolha correta das mídias a serem utilizadas, bem como a instrução dos alunos para utilização desses recursos.

Segundo Spagnolo e Mantovani $^{9}$, os educandos que já estão imersos no contexto do hibridismo tecnológico digital exigiam uma educação comprometida com mudanças e transformações evolutivas, as quais requerem ações que iriam além do espaço da sala de aula.

Maggio et al. ${ }^{10}$ argumentam que a adição de novas tecnologias para melhorar ou substituir as tradicionais na educação odontológica possuem modalidades que atraem e envolvem esta nova geração de alunos de maneira mais eficaz.

O presente trabalho tem por objetivo descrever a elaboração de uma plataforma multimídia para alunos de graduação e pósgraduação sobre a gênese dental e as possíveis alterações dento-alveolares que surgem no curso do desenvolvimento formativo do germe dentário, de maneira interdisciplinar, para que o discente de odontologia obtenha esse conhecimento através de uma visão ampliada e dinâmica, como fundamento para compreensão, da embriologia, fisiologia, patologia, radiologia e da clínica.

\section{METODOLOGIA}

Este estudo do tipo observacional foi aprovado pelo Comitê de Ética em pesquisa da Escola Bahiana de Medicina e Saúde Pública 
(EBMSP) (871.826) e encontra-se sob proteção intelectual junto ao Núcleo de Inovação Tecnológica (NIT) da instituição.

Um roteiro sistemático foi elaborado com a finalidade de orientar as etapas do trabalho, por meio de planejamento estruturado, baseado nas metas de aquisição para o banco de dados.

\subsection{Aquisição de Conteúdos}

Para elaboração do conteúdo didático da plataforma foram realizadas pesquisas bibliográficas em livros de histologia, radiologia, patologia, anatomia, fisiologia e odontopediatria, que servem como base no ensino da odontologia, além de buscas nas bases de dados Scielo, PubMed, Periódicos CAPES e em fontes de busca, como o Google Acadêmico. Dentre os artigos selecionados valorizou-se, a possibilidade da integralização de conhecimentos, se deu a partir do trabalho desenvolvido em 1960 por Carmem Nolla ${ }^{11}$.

Em seguida, o conteúdo recolhido foi transcrito para o Microsoft Word - Windows 2010 (Microsoft, Redmond, Washington, EUA) e, posteriormente resumido e transportado para o Microsoft PowerPoint - Windows 2010 (Microsoft, Redmond, Washington, EUA), para elaboração do protótipo inicial do software.

\subsection{Aquisição das imagens}

As imagens para ilustração dos casos clínicos (anomalias dentárias, estágios radiográficos da mineralização dental, tumores e cistos odontogênicos e doenças sistêmicas associadas a distúrbios do desenvolvimento dentário) do objeto em estudo foram selecionadas a partir dos arquivos de casos clínicos dos docentes de odontologia da EBMSP e do banco de dados do Instituto Baiano de Radiodiagnóstico e Bioimagem Odontológico (IBBRO).

Baseados nas Resoluções CNS 466/12 e 196/96, o coordenador do curso de Odontologia da EBMSP e o responsável técnico da clínica IBBRO, emitiram uma declaração de consentimento permitindo o uso de arquivos e similares dos prontuários. As imagens foram submetidas a processamento, seja por embaçamento, aplicação de tarjas ou recobrimento de identificações, para que fosse preservada integralmente a identidade dos pacientes dos quais foram obtidas.

As imagens histológicas, por sua vez, foram solicitadas ao grupo de Patologia e Histologia da EBMSp, mediante apresentação do objetivo do projeto, respeitando todas as normas estabelecidas pelo grupo.

Em seguida as imagens foram arquivadas no banco de dados da pesquisa e transportadas para o Microsoft PowerPoint Windows 2010 (Microsoft, Redmond, Washington, EUA), para ilustração do conteúdo transcrito.

\subsection{Desenvolvimento do programa}

Depois de confeccionado o protótipo inicial no Microsoft PowerPoint - Windows 2010 (Microsoft, Redmond, Washington, EUA), o modelo foi encaminhado para o Engenheiro de Computação, para elaboração do produto, no Flash + o ActionScript 3 (AS3) na plataforma Adobe AIR.

\subsection{Riscos}

O presente estudo não apresentou riscos para pacientes, pois não existe nenhuma intervenção nos mesmos. Os riscos jurídicos às instituições envolvidas quanto à utilização das imagens foram anulados pelo processamento das imagens, no sentido da preservação da identidade dos pacientes.

\subsection{Benefícios}

Os benefícios para os participantes da pesquisa são indiretos. Os mesmos contribuíram para a reformulação didática curricular no ensino da odontologia, participando assim do crescimento científico 
dos discentes da graduação e da pós-graduação. Além disso, contribuíram para a formação de uma visão mais generalista e interdisciplinar dos novos cirurgiões dentistas, alcançadas pela elaboração deste projeto de cunho multidisciplinar.

\section{APRESENTAÇÃO DO PROTÓTIPO DA PLATAFORMA}

A plataforma desenvolvida, intitulada
Ambiente Virtual de Ensino Odontológico (AVEO), apresenta características que serão aperfeiçoadas para o modelo final em virtude de se tratar de um protótipo.

Ao abrir o programa o discente se depara com o menu do objeto, o qual possui uma barra contendo os botões de acesso para a navegação e conhecimento científico das bases fundamentais para compreensão da gênese dentária (figura 1).

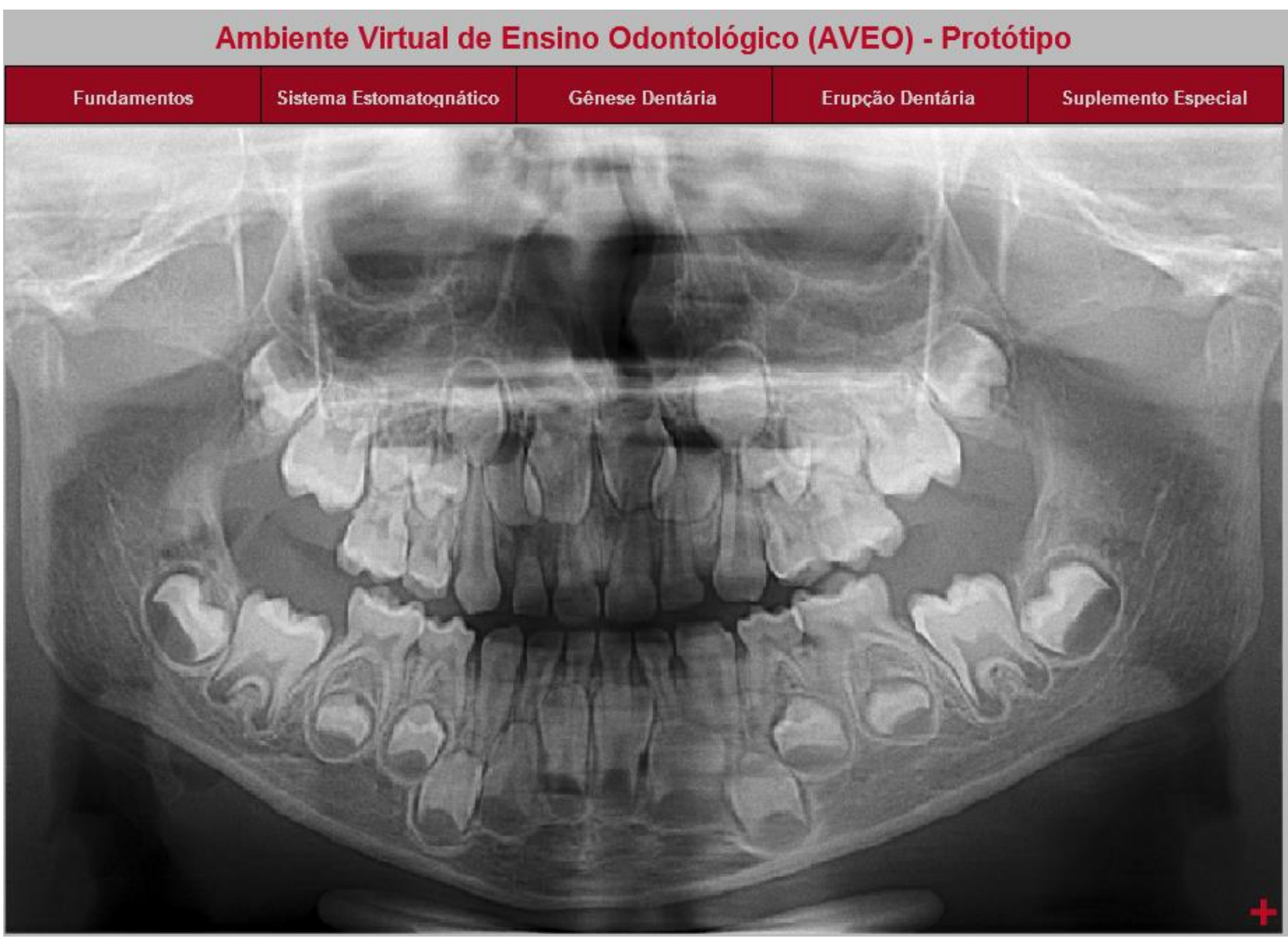

Figura 1 - Menu inicial do programa Ambiente Virtual de Ensino Odontológico (AVEO).

Ao clicar em fundamentos, o discente obterá todas as informações básicas sobre o projeto, indicações sobre a melhor maneira de utilizá-lo e tornar o estudo mais proveitoso.

Além disso, neste segmento, o aluno terá todo fundamento básico necessário para a compreensão dos termos adotados no estudo, das fases da gênese propriamente dita, a exemplo dos termos radiográficos radiolucidez e radiopacidade - , de modo a facilitar a assimilação para aqueles alunos que ainda estão iniciando a graduação em odontologia.

O botão correspondente ao Sistema Estomatognático tem por finalidade introduzir o aluno ao conhecimento sobre a formação embriológica e histológica, bem como as características radiográficas da maxila e mandíbula, de modo a garantir aos mesmos a base fundamental para compreensão dos 15(3):69-79, 2015. 
estágios que antecedem ou acontecem simultaneamente ao desenvolvimento do órgão dentário.
No segmento Gênese Dentária, encontram-se os links para navegação em todos os estágios da formação do dente (figura 2).

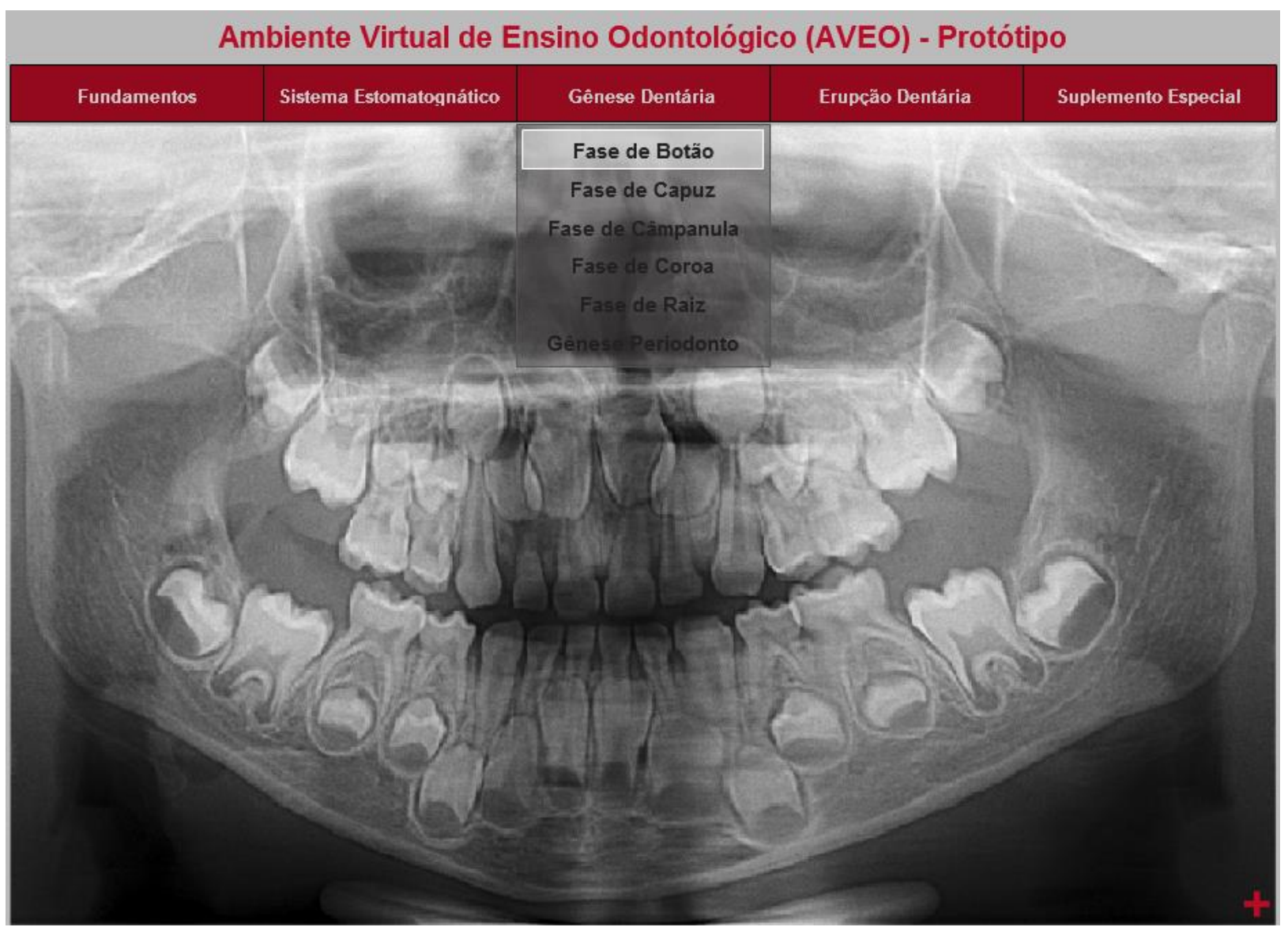

Figura 2 - Segmento Gênese Dentária: estágios de formação.

Ao clicar em alguma das fases, o usuário encontrará inicialmente o corte histológico correspondente ao estágio em questão, bem como botões onde o mesmo determinará o seu próprio mecanismo de aprendizado.

Desta forma, o objeto permite autonomia e direcionamento do estudo exclusivamente ao discente.

As figuras 3 a 6 exemplificam essa dinâmica, de modo que as informações só são expostas caso o estudante assim o deseje.

Os segmentos sobre a gênese dentária oportunizam a navegação em todos os desvios de normalidade correspondentes à fase, contemplando a interdisciplinaridade necessária para formação da sensibilidade diagnóstica.

Além da exposição das etapas da organogênese dentária, o software permite ao aluno acessar informações básicas sobre síndromes e desordens sistêmicas comumente associadas aos distúrbios do desenvolvimento do dente, bem como as características gerais e odontológicas de cada uma delas, contribuindo assim para a formação de um profissional generalista. 


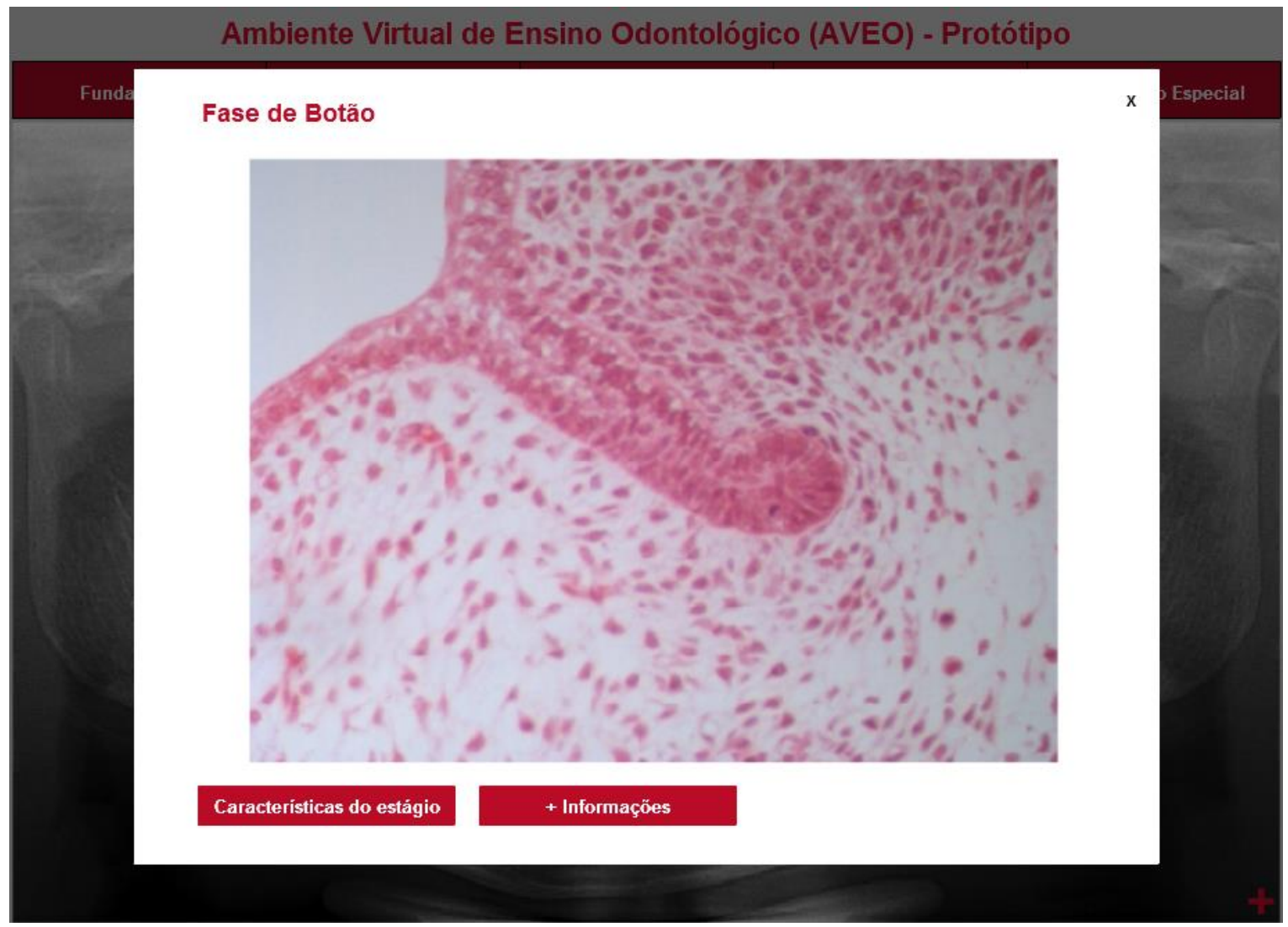

Figura 3 - Abertura da fase de Botão - corte histológico.

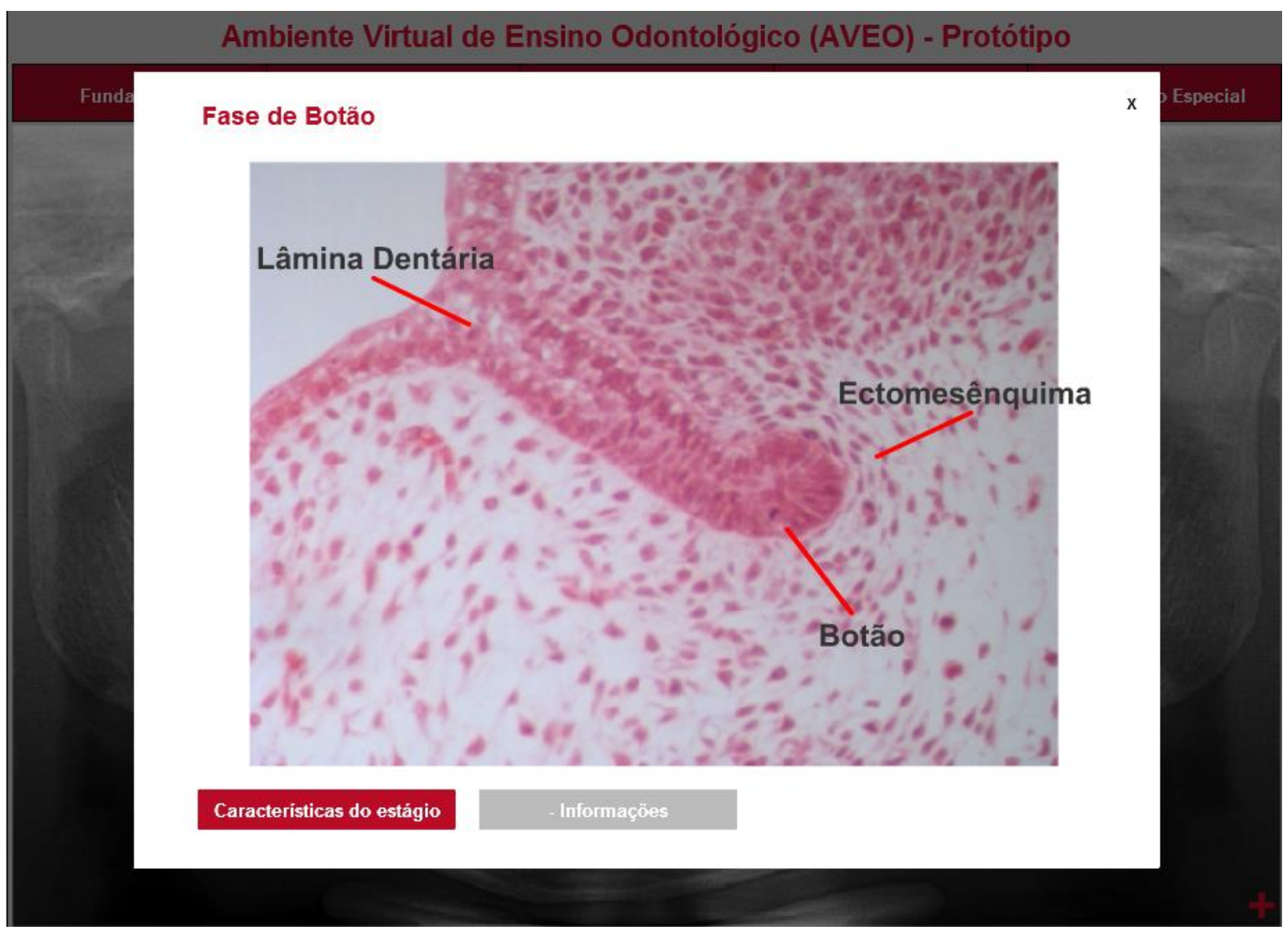

Figura 4 - Informações histológicas do corte da fase de Botão. 


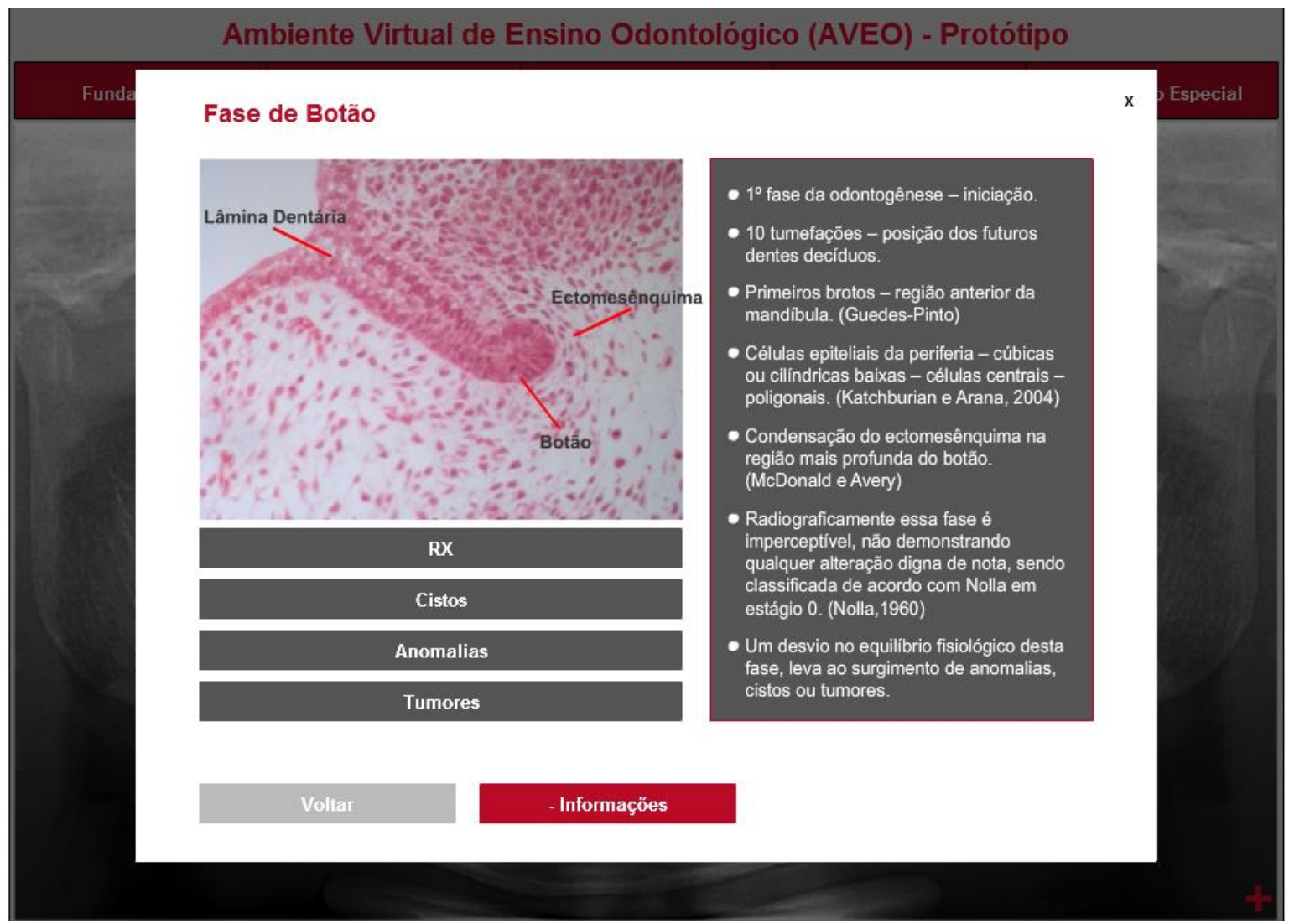

Figura 5 - Características do estágio de Botão, embasamento científico.

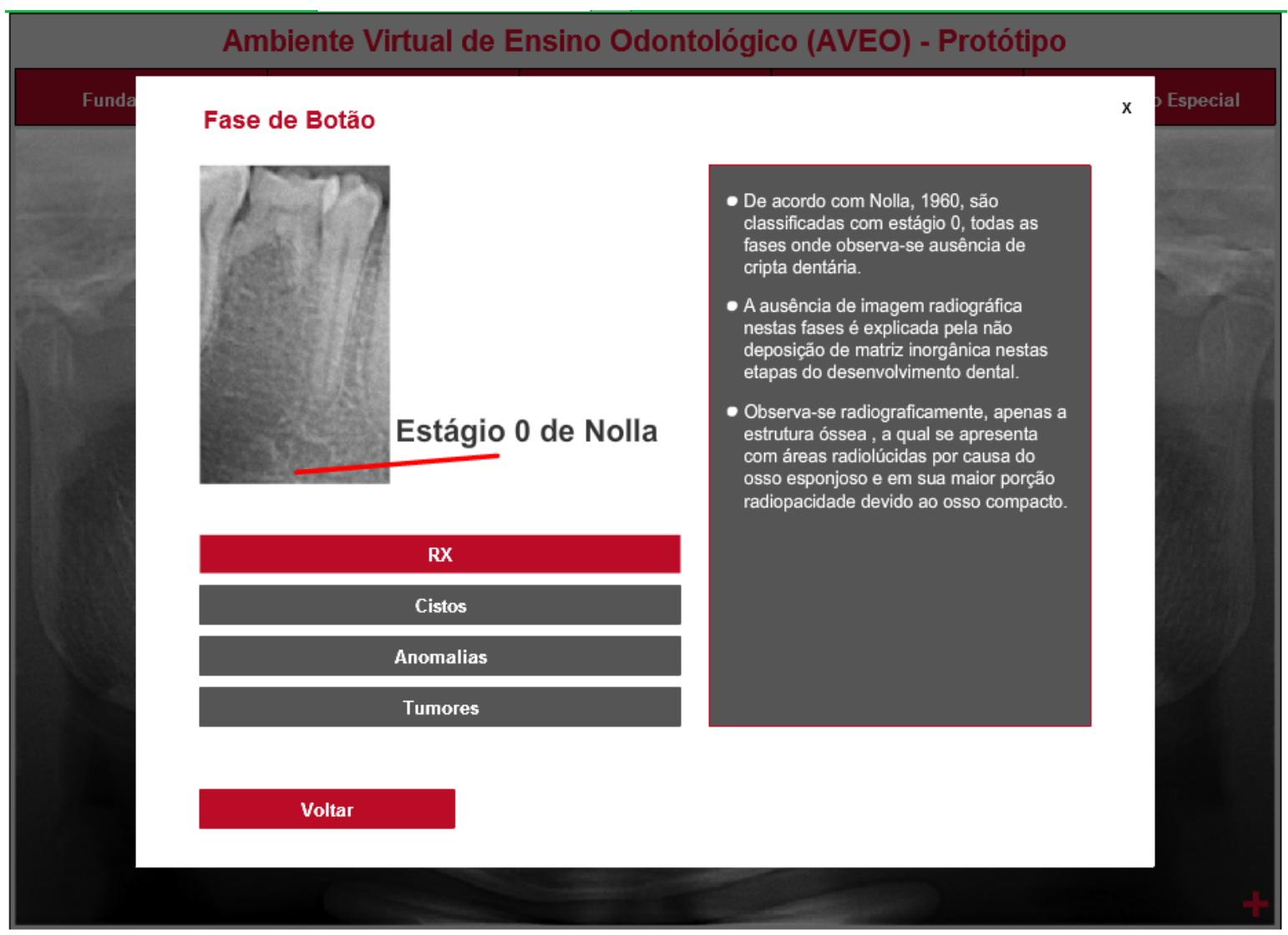

Figura 6 - Imagem radiográfica correspondente à fase de Botão. 


\section{DISCUSSÃO}

A educação vem sofrendo interferências positivas dos sistemas de inovação tecnológica nas últimas décadas, proporcionando ao educando e ao docente oportunidades variadas de propagação do conhecimento, emergindo, desta forma, a necessidade no ensino superior de desenvolver mecanismos digitais para se adequar ao novo processo de ensino aprendizagem.

Um estudo realizado por Guimarães et al. ${ }^{12}$ sobre a produção de material didático digital em comparação aos recursos tradicionais permitiu verificar que até mesmo os dispositivos móveis, de uso tão crescente entre os jovens, podem se tornar aliados à educação. Em virtude deste contexto, torna-se necessária a existência de materiais digitais com conteúdos de boa qualidade, permitindo ao aluno o acesso a informações úteis para seu crescimento profissional.

$\mathrm{Na}$ odontologia, o uso das tecnologias de comunicação e informação têm tido um crescimento exponencial, permitindo aos alunos o desenvolvimento da capacidade de procurar e selecionar informações, possibilitando assim a aprendizagem de uma maneira independente ${ }^{13}$.

Uma revisão da literatura desenvolvida por Jwayyed et al. ${ }^{14}$ sobre as técnicas, métodos, frequência e eficácia da educação assistida por tecnologia, comparando o ganho de conhecimento em educação assistida por tecnologia aos métodos tradicionais de ensino, encontraram que $67 \%$ dos estudos demonstraram a tecnologia apresentou resultados similares ou superiores ao método tradicional.

Dentro deste mesmo contexto outros trabalhos como o de Maggio et al. ${ }^{10}$, Vuchkova et al. ${ }^{15}$, Mitov et al. ${ }^{16}$ e Boynton et al. ${ }^{17}$, perceberam a melhora na assimilação dos conteúdos quando os recursos tecnológicos eram integrados ao currículo tradicional.
Apesar da aceitação e da dinamização proporcionada pelos recursos inovadores, Maggio et al. ${ }^{10}$ observaram que os alunos não desejam a substituição total do método tradicional, optando pelo entrelaçamento dos dois modelos de ensino.

Este posicionamento sustenta um dos princípios estabelecidos neste estudo, onde o software desenvolvido surge como um complemento facilitador ao modelo convencional, através do seu caráter incitativo, conduzindo o aluno a explorar sua curiosidade pelo conteúdo, tendo o professor como figura relevante neste processo, não mais como um agente transmissor de informações e sim como um tutor, mediando o processo de aprendizagem.

Ainda que comprovado o potencial facilitador das hipermídias e dos demais recursos de tecnologia da informação e comunicação, o estudo bibliométrico desenvolvido por Cruz et al. ${ }^{18}$ revelou um número pequeno de publicações sobre o uso desses recursos pedagógicos em saúde no Brasil, o que levanta alguns questionamentos sobre a baixa utilização destas ferramentas no país e sobre o pouco interesse dos autores sobre o tema.

Durante a construção deste estudo, observou-se que no campo odontológico esta realidade foi bem marcante. Isto também foi percebido por Ávila ${ }^{19}$, que observou em seu levantamento bibliográfico o baixo emprego do ensino assistido por computadores em Odontologia, denotando demora dessa área em aderir à informática no ensino.

Todavia, os trabalhos na área odontológica encontrados para esta pesquisa revelam em seus resultados índices elevados de sucesso, quando os mesmos são aplicados e selecionados de maneira adequada, sendo a maioria deles destinados ao ensino da radiologia, da anatomia e da morfologia dentária. 
Como exemplos de softwares com finalidade no ensino e capacitação da Odontologia surgem, o software de Visualização em 3D desenvolvido por Vuchkova et al. ${ }^{15}$, que tem por objetivo o ensino da interpretação radiográfica das patologias bucais; o MorphoDent desen-volvido por Mitov et al. ${ }^{16}$ relacionado ao ensino da anatomia da dentição adulta; e o Interactive Histology Software (IHS) desenvolvido por Rosas et al. ${ }^{20}$, que direciona o ensino da histologia com imagens em alta resolução, associada a rótulos com textos informativos.

Contudo, nenhum destes softwares, nem em outros trabalhos similares encontrados durante o levantamento bibliográfico trazem a ideia da interdisciplinaridade como proposto pelo software desenvolvido neste estudo, que teve por objetivo a integração dos conteúdos, evitando a sua segmentação, como é de costume do método tradicional de ensino na graduação odontológica. Desta forma, concede-se aos discentes oportunidades para exploração do senso crítico, base científica para elaboração das etapas processuais do diagnóstico e consequente planejamento para o tratamento.

A escolha por esta metodologia se deu baseada nos achados de Baghdady et al. ${ }^{21}$, que observaram a melhora na construção do conhecimento e maior produção da acurácia diagnóstica, quando a ciência básica, a fisiopatologia, as características clínicas e radiográficas são integradas e simultaneamente associadas ao conteúdo abordado.

De acordo com Arruda et al. ${ }^{22}$, a necessidade de fornecer ao aluno o conceito integral da profissão é imperiosa, tendo como alvo a formação de clínicos gerais aptos a diagnosticar, planejar, prognosticar, executar e avaliar os planos de tratamentos odontológicos integrados. O que reafirma o intuito do estudo em agregar os componentes curriculares.

No que tange ao desenho e dinâmica do software, este estudo desenvolveu o conteúdo de forma a permitir com que o discente obtivesse o material didático complementado com animações, imagens clínicas e radiográficas, de modo a dinamizar o processo de aprendizagem, através de uma plataforma simples e de fácil execução.

Conforme Woelber et al. $^{23}$, os programas de software com finalidade de ensino não precisam ser excessivamente trabalhosos. Os autores verificaram que mesmo softwares simples têm potencial benéfico na educação odontológica, sendo de grande aceitação pelos acadêmicos.

O desenvolvimento dentário foi o tema escolhido para compor o conteúdo didático deste estudo, pelo fato do mesmo ser o fundamento para o estudo nas inúmeras áreas do conhecimento da Odontologia. Os estágios da organogênese dental, mais precisamente a fase de mineralização dos dentes decíduos segundo McDonald e Avery ${ }^{24}$, tem uma extrema relevância clínica para o dentista, tendo em vista que frequentemente, a necessidade de explicar aos pais o processo de desenvolvimento e mineralização, ou em breves discussões da morfologia dos dentes decíduos antes de serem considerados os procedimentos restauradores.

Embora o software ainda se encontre em construção, este estudo nos estimula a prosseguir de modo a propiciar ao discente um programa com amplo conteúdo didático, animações aperfeiçoadas e agregação dos componentes avaliativos clínicos.

\section{CONSIDERAÇÕES FINAIS}

A plataforma em questão, mesmo sendo um projeto piloto, já demonstra total potencialidade para o ensino, por conseguir integrar todas as informações concernentes à gênese dentária, contribuindo, assim, para o estímulo do auto aprendizado, exploração do senso crítico, e ampliação do conhecimento dos discentes, favorecendo o seu desenvolvimento 
profissional.

\section{REFERÊNCIAS}

1. Lopes AC, Buratto AP. Desenvolvimento de um software educacional e sua aplicação no ensino de química. [Trabalho de Conclusão de Curso]. Pato Branco (PR): Universidade Tecnológica Federal do Paraná; 2011.

2. Melo JRF. A formação inicial do professor de química e o uso das novas tecnologias para o ensino: Um olhar através de suas necessidades formativas. [Dissertação]. Natal ( $R N)$ : Universidade Federal do Rio Grande do Norte; 2007.

3. Machado LL, Silva JT. Objeto de aprendizagem digital para auxiliar o processo de ensino-aprendizagem no ensino técnico em informática. Renole 2005; 3(2): 1-16.

4. Haguenauer CJ, Lopez FB, Martins FN. Estudo comparativo de ambientes virtuais de aprendizagem. Rev Digital da CVA 2003; 2 (5):1-9.

5. Schmidt P. Digital learning programs competition for the classical microscope? GMS Z Med Ausbildung. 2013;30(1):1-11.

6. Vogel D, Klassen J. Technology-supported learning: status, issues and trends. J Comput Assist Lear 2001; 17:104-14.

7. Reynolds P. UDENTE (Universal Dental ELearning): a golden opportunity for dental education. Bull Group Int Rech Sci Stomatol Odontol 2012;50(3):11-9.

8. Niederhauser DS, Stoddart T. Teachers' instructional perspectives and use of educational software. Teach Teach Educ 2001;17:15-31.

9. Spagnolo C, Mantovani AM. Aprendizagem colaborativa na educação escolar: novas perspectivas para o processo de ensinar e aprender. Rev Digital da CVA 2013;8(30):110.

10. Maggio MP, Hariton-Gross K, Gluch J. The use of independent, interactive media for education in dental morphology. J Dent Educ 2012;76(11):1497-511.

11. Nolla CM. The devoelopment of the permanente teeth. J Dent Child 1960;27: 254-66.

12. Guimarães K, Lima ME, Pinheiro R, Sobrinho MC, Geller MTB. Produção de material didático digital no formato ePub. Computer on the Beach 2014;1:52-63. .

13. Fontanella V, Schardosim M, Lara MC. Tecnologias de informação e comunicação no ensino da Odontologia. Rev ABENO 2007;7(1):76-81.

14. Jwayyed S, Stiffler KA, Wilber ST, Southern A, Weigand J, Bare R, et al. Technology-assisted education in graduate medical education: a review of the literature. Int J of Emerg Med 2011;4:51-63.

15. Vuchkova J, Maybury TS, Farah CS. Testing the educational potential of 3D visualization software in oral radiographic interpretation. J Dent Educ 2011;75(11): 1417- 425.

16. Mitov G, Dillschneider T, Abed MR, Hohenberg G, Pospiech P. Introducing and evaluating MorphoDent, a web-based learning program in dental morphology. $\mathrm{J}$ Dent Educ 2010;74(10):1133- 39.

17. Boynton JR, Green TG, Johnson LA, Nainar SMH, Straffon LH. The virtual child: evaluation of an internet-based pediatric behavior management simulation. J Dent Educ 2007;71(9):1187-93.

18. Cruz DI, Paulo RRD, Dias WS, Martins VF, Gandolfi PE. O uso das mídias digitais na educação em saúde. Cad FUCAMP 2011;13(10):130-42.

19. Ávila MAG. Software anatomia em radiografias panorâmicas: Avaliação do método de ensino-aprendizado em Odontologia. [Dissertação]. São Paulo (SP): Faculdade de Odontologia da Universidade de São Paulo; 2004.

20. Rosas C, Rubí R, Donoso M, Uribe S. Dental students' evaluations of an interactive 
histology software. J Dent Educ 2012;76(11):1491-96.

21. Baghdady MT, Carnahan H, Lam EWN, Woods NN. Integration of basic sciences and clinical sciences in oral radiology education for dental students. J Dent Educ 2013;77(6):757-63.

22. Arruda WB, Siviero M, Soares MS, Costa CG, Tortamano IP. Clínica integrada: o desafio da integração multidisciplinar em Odontologia. RFO 2009; 14(1):51-5.

23. Woelber JP, Hilbert TS, Ratka-Kruger P. Can easy-to-use software deliver effective elearning in dental education? A randomised controlled study. Eur J Dent Educ 2012;16:187-92.

24. McDonald RE, Avery DR. Desenvolvimento e morfologia dos dentes decíduos. In: McDonald RE, Avery DR. Odontopediatria. 7ed. Rio de Janeiro: Guanabara Koogan; 2001 :37-42.

\footnotetext{
ABSTRACT

A software development as a didactic object for understanding the dental genesis events

Technology has gradually spread over all fields of society, becoming indispensable and promoting evolution. The same way it has opened a completely new world of possibilities for the development of knowledge in education. This work presents a new software conceived as a tool for either undergraduate or graduate dental students to provide them a thorough, dynamic and interdisciplinary vision of dental
}

genesis, as well as the possible changes in dentoalveolar structures during the developmental process of teeth germs. It referred to scientific material from books adopted in Dentistry education, articles from Scielo, Pubmed and Periodicals CAPES databases and other information accessed by search tools such as Google Scholar and similar ones. The dynamic process was accomplished by using the Flash + Action Script 3 (AS3) system in Adobe AIR platform, allowing students to navigate throughout all the developmental phases of teeth germs by clicking on desired file. The interdisciplinarity and interactivity of information made possible with this platform allows students to build up highly importance knowledge in Dentistry, as it works as a didactic instrument in accordance to the new teaching tendency, which will consequently help in diagnostic reasoning.

Descriptors: Education. Software. Dentistry. Technology. Learning. Anomaly.

Correspondência para:

Fellipe Moraes

e-mail: fellipempf@gmail.com

Rua Banco dos Ingleses, 1/102

40080-040 - Salvador - BA 